\title{
The mucopolysaccharidoses: a clinical review and guide to management
}

\author{
J E Wraith
}

The mucopolysaccharidoses are a group of inherited metabolic disorders caused by a deficiency of specific lysosomal enzymes. The enzyme deficiency results in interference with cellular function because of excess accumulation within the cells of partially degraded glycosaminoglycans (GAGS), which are also excreted to excess in the urine of affected patients.

Although the mucopolysaccharidoses were first described clinically by Hunter in 1917 , their biochemical basis was not fully elucidated until the 1950 s and 1960 s. More recently the molecular biology of many of the subtypes has been described. ${ }^{1}$

The ubiquitous nature of GAGS within the connective tissue of the body results in a wide range of clinical effects. The type of GAG stored depends on the specific enzyme deficiency and classification of the disorders is now based upon these deficiencies, rather than clinical features. The table outlines present day classification with details of the accumulating compounds and enzyme deficiencies, as well as the eponym used for each condition. Excellent accounts of the biochemical basis of the mucopolysaccharidoses are available and this area will not be considered further.

In keeping with many other multisystem disorders affected patients are often under the care of many different clinicians and coordination of care can be difficult. A special clinic for children with mucopolysaccharidoses has been established in the Willink Biochemical Genetics Unit and this review outlines important management issues that occur with each

Correspondence to: Dr Wraith. particular subtype. The information is based on the clinical details of 225 patients who have attended this clinic from the UK and abroad. A breakdown of the patients has been appended to the table.

\section{General considerations}

A multidisciplinary approach to management is necessary. Paediatric subspecialties, such as cardiology, anaesthesia, orthopaedics, otorhinolaryngology, ophthalmology, and neurosurgery, as well as many paramedical groups: for example physiotherapy, occupational therapy, audiology, speech therapy and psychology, will all have an important input. Diagnosis and evaluation is best undertaken in a major centre. The creation of our special clinic has allowed colleagues in the above related specialties to become highly experienced in the management of complications associated with the mucopolysaccharidoses. After assessment and counselling the subsequent care of the children can often be shared with the local paediatrician.

\section{Regular assessments}

As with many chronic progressive disorders the child has to be seen frequently enough to allow recognition of potential problems, leaving the parents free to enjoy the periods of better health. An annual assessment at the mucopolysaccharidoses clinic has proved valuable. Enough time is set aside to allow parents to express concerns and anxieties often not expressed in subspecialty clinics. Often a short stay in hospital can incorporate reviews by a number of subspecialists and in this way the progress of the disease can be monitored and complications recognised and treated early.

\section{Anaesthesia}

A number of reviews have highlighted the difficulties associated with this group of disorders. $^{2-4}$ A careful preoperative and perioperative management plan is required if disaster is to be averted. Intubation is often extremely difficult, although this has been helped by the use of fibreoptic laryngoscopy and bronchoscopy. In difficult cases the laryngeal mask airway has proved to be a useful additional aid. Anaesthesia must be

${ }^{\star} \mathrm{DS}=$ dermatan sulphate, $\mathrm{HS}=$ heparan sulphate, $\mathrm{KS}=$ keratan sulphate, $\mathrm{CH}=$ chondroitin sulphate. 
undertaken only by anaesthetists expert in maintaining paediatric airways and in centres with adequate intensive care facilities. In the severely affected patient operations should only be performed for life threatening complications or in an effort to greatly improve the child's quality of life. Minor or cosmetic procedures should not be undertaken or should be performed under local anaesthesia.

\section{Eponyms, heterogeneity, and phenotype}

The mucopolysaccharidoses are most commonly known by their eponomous titles, as outlined in the table. However, as our understanding has increased it has become increasingly clear that these are a gross simplification. Most commonly the disorders are divided into 'mild' and 'severe' variants. For example, in MPS I the severe variant is known as Hurler's syndrome and the mild variant Scheie's syndrome. Anything apparently not conforming to this division is called Hurler/Scheie syndrome by most paediatricians. It is now apparent, like most genetic disorders, that there is a continuous spectrum of phenotype from the very severe to the most mildly affected. Genotype analysis, where known, in this group of disorders has confirmed that many different mutations are responsible for these phenotypic differences. It is preferable now to speak in terms of enzyme deficiency rather than eponym, but as the latter are so well established it will take time for this to achieve universal acceptance.

\section{Presenting features}

Patients with a mucopolysaccharidosis usually present in one of three ways: (i) as a dysmorphic syndrome, for example MPS I, MPS II, MPS VII; (ii) with severe behavioural distur-

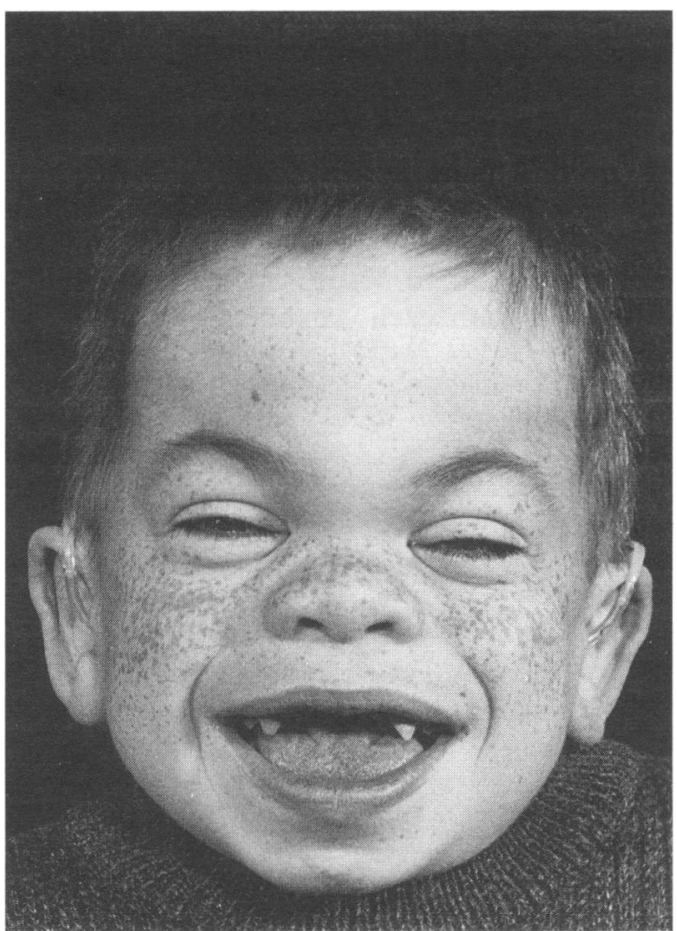

Figure 1 Typical facial features of Hurler's syndrome. bance and dementia, for example MPS III; and (iii) with evidence of a severe bone dysplasia, moderate dysmorphism, and normal intelligence, for example MPS IV, MPS VI.

\section{Particular problems associated with each enzyme deficiency}

MUCOPOLYSACCHARIDOSIS TYPE I, IDURONIDASE DEFICIENCY (MPS IH, MPS IS, MPS IH/S, HURLER'S SYNDROME, SCHEIE'S SYNDROME AND OTHER VARIANTS)

Fifty seven patients with iduronidase deficiency have been assessed in the unit and the full clinical spectrum ranging from death in early infancy due to cardiomyopathy to near normal adult patients has been observed. The commonest phenotype is that of 'classical' Hurler's syndrome and a number of these patients have been found to be homozygous for a recently described common mutation in the iduronidase gene. ${ }^{5}$ The characteristic dysmorphism associated with this disorder is familiar to all paediatricians (fig 1).

In the severely affected patient the clinical course is dominated by airway problems and frequent upper and lower respiratory infections are common. Diagnosis should be followed by immediate referral to the ear, nose, and throat clinic as all affected children require treatment for upper airways obstruction and middle ear disease. Obstructive sleep apnoea is an almost universal finding. Initially this can be helped by tonsillectomy and adenoidectomy, but many patients require nocturnal oxygen treatment later in the course of the illness.

Cardiac disease is very common and presentation and early death from cardiomyopathy is a recognised complication of this disease. ${ }^{6}$ In other patients asymmetrical ventricular septal hypertrophy is a frequent finding early in the disorder and is often followed by variable thickening of the mitral and aortic valves. Coronary insufficiency is known to occur and sudden death from arrythmia has been assumed in some patients.

Despite these potentially severe physical problems early intellectual development is usually normal and affected patients do not have severe learning difficulties until much later in the course of the disease. The majority of children attain some social skills and many are capable of starting normal nursery schools. Mobility may be limited by joint stiffness which is progressive, but usually not painful, and by the protuberant abdomen due to hepatosplenomegaly. The latter is also probably responsible for the high incidence of umbilical and inguinal hernias seen in these patients.

Hydrocephalus requiring shunt insertion was noted in six patients. If ignored the head can reach enormous proportions and make nursing of the child difficult in the latter stages of the illness.

Corneal clouding can be very variable. Glaucoma is often quoted as a complication of MPS I, but was seen in only one patient in this series. Sudden blindness occurred in one patient with no apparent cause, electroretinograms were normal, but visual evoked 


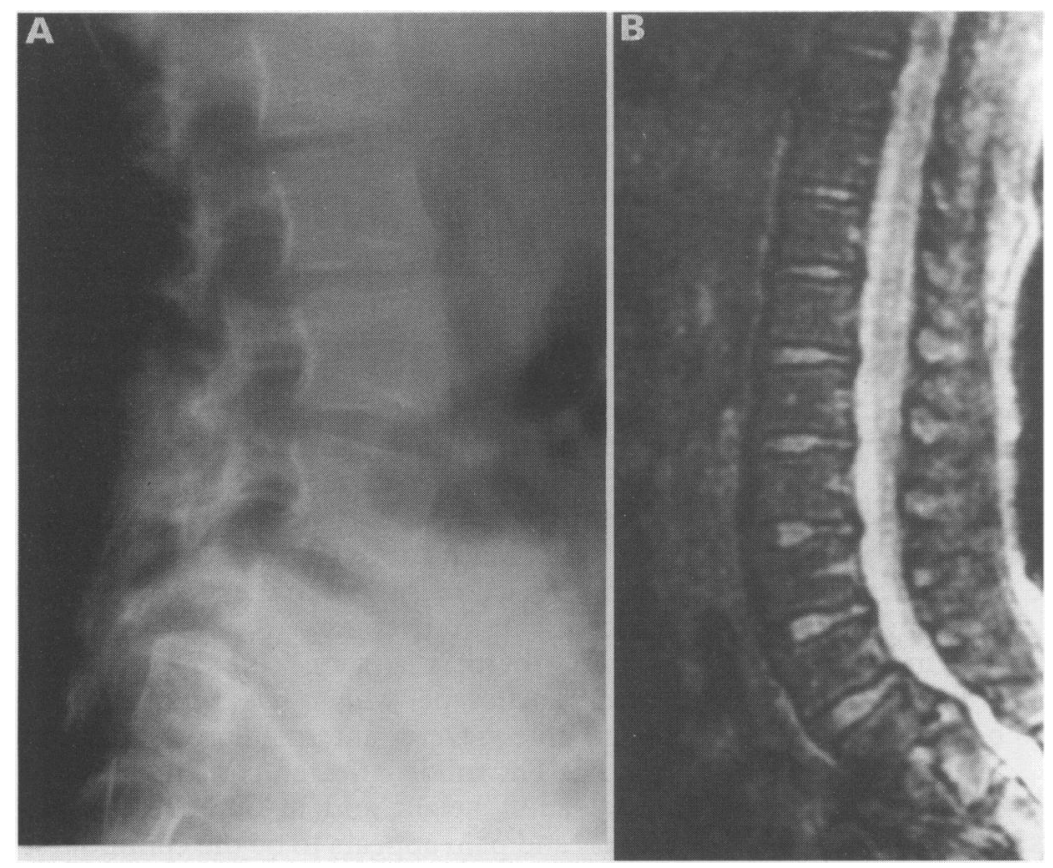

Figure $2 A$ Radiograph of the lumbar-sacral spine in a patient with MPS IS showing the typical lumbar-sacral spondylolisthesis. (B) Magnetic resonance image of the same patient showing narrowing and compression of the spinal canal at the level of the spondylolisthesis.

responses absent. Magnetic resonance imaging of the visual pathways was normal.

Death usually occurs in the first decade of life and is most often due to cardiorespiratory failure.

In the adult patient with iduronidase deficiency, the dysmorphic features are often very mild and there are different priorities with regard to management. The clinical picture is usually dominated by the orthopaedic complications of the disease. Poor hand function, partly due to chronic carpal tunnel syndrome is extremely common. ${ }^{7}$ Progressive joint stiffness and severe back pain are usual. Radiography of the lower lumbar spine has shown a very high incidence of spondylolisthesis in these patients and in some this has been associated with spinal cord compression (fig $1 \mathrm{~A}$ and $2 \mathrm{~B}$ ). As the patient gets older corneal clouding may interfere with vision and corneal grafting has been performed on two patients. In these circumstances it is essential to confirm that the loss of vision is not due to retinal disease.

In older patients mitral and/or aortic incompetence are common.

The oldest patient seen in the clinic is currently 48 years of age: she shares the same genetic mutations as Scheie's original patient. ${ }^{8}$

MUCOPOLYSACCHARIDOSIS TYPE II, IDURONATE SULPHATE SULPHATASE DEFICIENCY (MPS TYPE II, HUNTER'S SYNDROME)

Forty six patients with this variant have been reviewed. The vast majority of patients have been severely affected (35), but again a spectrum of clinical effect can be observed. Severely affected patients share many of the features of classic Hurler's syndrome, but tend to be milder and have less severe skeletal involvement. The classic nodular rash said to be pathognomic of this disorder is rare and has been observed in only two patients. Once again ear, nose, and throat and airway problems dominate the clinical course. In addition most patients are prone to severe bouts of diarrhoea, the cause of which remains obscure. In addition we have observed other unusual manifestations of gastrointestinal dysfunction not previously appreciated, such as, spontaneous perforation of the stomach and intestinal pseudo-obstruction.

Progressive neurodegeneration results in a vegetative existence for most patients from early teenage years with death around the age of 15-16 years.

In mildly affected patients cervical myelopathy due to dural hyperplasia and thickening of the ligamentum flavum is probably inevitable. In patients with declining exercise tolerance or upper motor neurone signs in the limbs, magnetic resonance imaging of the craniocervical junction should be performed. At the same time the airway can be imaged and an assessment made of the inevitable anaesthetic problems in this group. No operative procedure should be performed on a mildly affected MPS II patient until it has been established whether or not cervical cord compression is present. The manipulation involved in a difficult anaesthetic can lead to sudden cord compromise and result in quadriplegia.

It is interesting to note that even in mildly affected patients with normal intellect that imaging of the central nervous system is often grossly abnormal (fig 3).

The genetics of this mucopolysaccharidosis differ from the others as the disorder is inherited as an X linked recessive. Only a minority of affected boys have a demonstrable deletion in their iduronate sulphatase gene $(20 \%)$, the

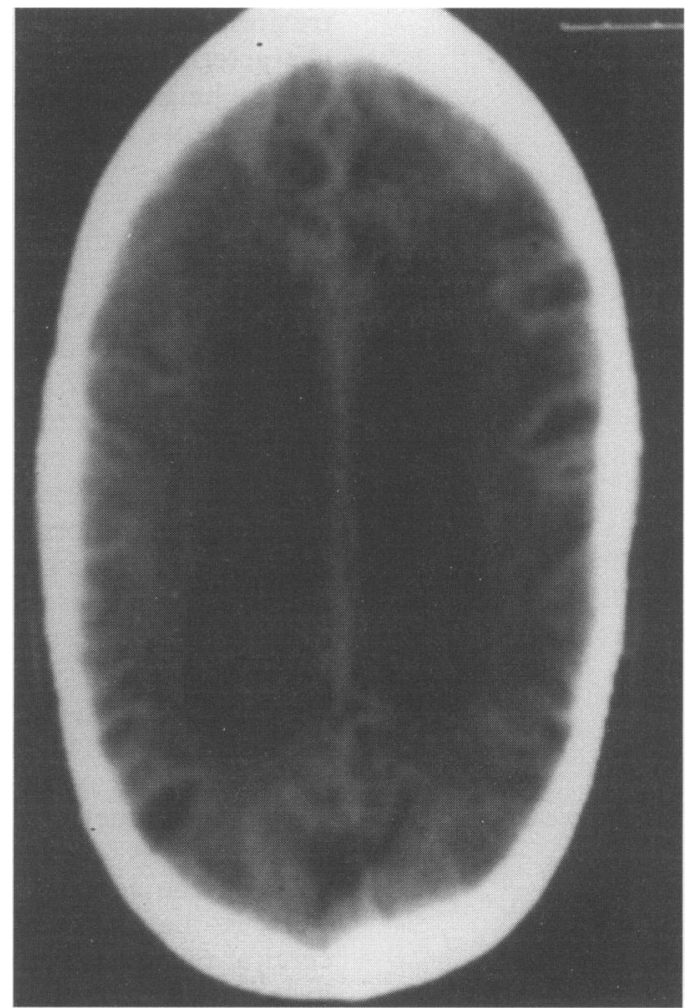

Figure 3 Computed tomogram of an intellectually normal man aged 24 years with MPS II. Note the ventricular dilatation and 'cyst-like structures' in the cerebral matter. 
majority of defects being various point mutations. ${ }^{9}$

MUCOPOLYSACCHARIDOSIS TYPE III (MPS IIIA, IIIB, IIIC, IIID, SANFILIPPO'S SYNDROME)

In this disorder four enzyme defects are known to cause an identical clinical phenotype. This is the commonest of the mucopolysaccharidoses in the UK and its management has been reviewed recently. ${ }^{10}$ Seventy nine patients with MPS III have been assessed. Again, heterogeneity produces a variable clinical phenotype and a spectrum of effect is noted. Some patients are very mildly affected and it may be that this disorder is underdiagnosed. Mild dysmorphic features are combined with a very severe behavioural disturbance and the disorder is extremely difficult to manage.

The molecular basis of the commonest variant of MPS III is yet to be established.

MUCOPOLYSACCHARIDOSIS TYPE IV (MPS IVA, MPS IVB, MORQUIO'S SYNDROME)

No patients with MPS IVB have been seen in the unit; this appears to be a rare variant in the UK. Thirty eight patients with MPS IVA have been seen and assessed. Affected patients are of normal intelligence and the clinical picture is dominated by a severe bone dysplasia. Skeletal radiographs are often abnormal from birth, but diagnosis is usually established in the first year of life after parents notice the obvious spinal deformity associated with this disease.

Motor milestones are achieved at the normal times, but the gait becomes increasingly abnormal due to the development of severe genu valgum secondary to ligamentous laxity; this, combined with the increasing sternal protrusion and severe pes planus, leads to a very characteristic posture. In the severe form of the disease height prognosis is extremely poor and few patients will be taller than $100 \mathrm{~cm}$ as adults.

The major complication of this mucopolysaccharidosis is the development of neurological abnormality secondary to cervical myelopathy (also occurs in MPS I but is less common). This may be of sudden onset resulting in death after a simple fall as has occurred in two of our patients and a number of their siblings, not seen in the unit, or it may occur insidiously with a lack of exercise tolerance predating frank neurological abnormality. This complication must be looked for with vigour and we currently perform magnetic resonance imaging of the craniocervical junction on an annual basis in patients with MPS IV. It is our practice to perform prophylactic occipitocervical fusion in patients with instability. We do not wait for the onset of neurological abnormality. The instability is due to a combination of a hypoplastic, poorly ossified dens and ligamentous laxity in the cervical spine. What has become clear is that successful fusion in the upper cervical region is often followed by progressive instability further down the cervical spine and multiple fusions may be required in some patients. This complication is most commonly seen in patients who remain independently mobile after the procedure. In patients who are chair bound before surgery, sitting posture may lead to subluxation of vertebrae lower down the spinal column, for example in the mid-thoracic region.

Affected patients are not dysmorphic, but do have an increased incidence of ear, nose, and throat disease and in addition have characteristic teeth which are prone to dental caries. Fine corneal opacity is usual, but never as severe as patients with MPS I. Aortic incompetence is a usual finding in the adult patient. Life expectancy should be good if cervical complications are avoided. Some patients, however, develop progressive cardiorespiratory disease secondary to their restricted chest movement.

Again, there is a very wide spectrum of clinical phenotype which has been broadly divided into severe, intermediate, and mild MPS IV. The mildly affected patient can be of normal adult height and in our patients hip stiffness and pain appears to be the commonest mode of presentation.

As adults patients with MPS IV often develop aches and pains in various joints. We assume that this is 'wear and tear' accelerated by the abnormal joint posture. The symptoms respond variably to analgesia and non-steroidal anti-inflammatory agents.

It has been proposed that the prognosis for girls with this disorder is better than for boys and this was attributed to their less active lifestyle'. ${ }^{11}$ In our patients we can detect no difference in the severity of the disease between the sexes and the incidence of cervical cord problems is the same in both groups.

\section{MUCOPOLYSACCHARIDOSIS TYPE VI,} $N$-ACETYLGALACTOSAMINE-4-SULPHATASE DEFICIENCY (MPS VI, MAROTEAUX-LAMY SYNDROME)

This mucopolysaccharidosis appears to be very rare in the UK. Only five patients have attended for assessment and follow up. Again this is a heterogeneous disorder and is usually considered to the 'mild' because of the retention of normal IQ by affected patients. The disorder should not, however, be considered benign. In addition to relatively easily treated early complications such as ear, nose, and throat disease or carpal tunnel syndrome there are some more serious potential problems. Presentation with endocardial fibroelastosis ${ }^{12}$ or cardiomyopathy ${ }^{13}$ has been reported, and in addition cervical myelopathy is known to occur. ${ }^{14}$ The most serious complication, as there is no effective treatment, is the development of progressive, diffuse airway narrowing leading to cor pulmonale and death in late teens or early twenties.

In theory, MPS VI is the one mucopolysaccharidosis most suitable to 'curative' treatment by enzyme replacement, as the central nervous system is not affected. There is also an excellent feline animal model which makes assessment of such treatment appropriate before human trials. 
MUCOPOLYSACCHARIDOSIS TYPE VII, $\beta$-GLUCURONIDASE DEFICIENCY (MPS VII, SLY'S DISEASE)

This disorder is extremely rare and no affected patients have been seen. Indeed, in our laboratory where we receive annually over 1000 blood samples for lysosomal enzyme assay and over $\mathbf{5 0 0}$ urines for mucopolysaccharide analysis we have encountered this disorder on only two occasions in the last 10 years. The phenotype of affected patients is again very variable and ranges from presentation with hydrops fetalis to a relatively mild Hurler phenotype presenting in adult life.

\section{OTHER DISORDERS}

While not the purpose of this review it should be noted that there are a number of storage disorders which produce a phenotype not dissimilar to the mucopolysaccharidoses. In addition to the patients outlined above we have also seen and assessed 20 patients with mucolipidosis II and III, eight patients with mannosidosis ( $5 \alpha$ and $3 \beta$-mannosidase deficient), and one with sialic acid storage disease. All presenting initially as possible mucopolysaccharidosis patients.

\section{Discussion}

The mucopolysaccharidoses are a group of devastating disorders and parents need considerable support in dealing with an affected child. The mucopolysaccharidoses society ${ }^{\star}$ has offered many parents and affected individuals considerable help as well as raising significant sums of money for research.

A sound understanding of the phenotypic possibilities within each subgroup allows for a logical approach to management which is inevitably multidisciplinary. The paediatrician should play a lead part in this process and coordinate services for affected patients. The disorders should no longer be regarded as 'untreatable' as quality of life for many individuals can be greatly improved after relatively simple procedures.

Finally, it is important to remember that these are genetic disorders. First trimester prenatal diagnosis is possible for all of the subtypes. Thanks to Professor John Hopwood and colleagues, depart-
ment of chemical pathology, Adelaide Children's Hospital, for ment of chemical pathology, Adelaide

molecular analyses on many patients. `The Society for Mucopolysaccha
Avenue, Amersham, Bucks HP6 5BX.

1 Hopwood J, Morris CP. The mucopolysaccharidoses: diagnosis, molecular genetics and treatment. Mol Biol Med 1990; 7: 381-404

2 Belani KG, Krivit W, Carpenter BLM, et al. Children with mucopolysaccharidosis: perioperative care, morbidity, mortality, and new findings. I Pediatr Surg 1993; 28: 403-10.

3 Diaz JH, Belani KG. Perioperative management of children with mucopolysaccharidoses. Anesth Analg 1993; 778 1261-70.

4 Sjogren P, Pedersen T, Steinmetz H. Mucopolysaccharidoses and anaesthetic risks. Acta Anaesthesiol Scand 1987; 31: 214-8.

5 Scott HS, Nelson PV, Cooper A, Wraith JE, Hopwood J, Morris CP. Mucopolysaccharidosis type I (Hurler syndrome): linkage disequilibrium indicates the presence of a major allele. Hum Genet 1992; 88: 701-2.

6 Donaldson MDC, Pennock CA, Berry PJ, Duncan AW, Cowdrey $J E$, Leonard $J V$. Hurler syndrome with car diomyopathy in infancy. $\mathcal{F}$ Pediatr 1989; 114: 430-2.

7 Wraith JE, Alani SM. Carpal tunnel syndrome in the mucopolysaccharidoses and related disorders. Arch $D i$ Child 1990; 65: 962-3.

8 Scheie HG, Hambrick GW, Barnes LA. A newly diagnosed forme fruste of Hurler's disease (gargoylism). Am $g$ Ophthalmol 1962; 53: 753-69.

9 Bunge S, Steglich C, Zuther C, et al. Iduronate-2-sulfatase gene mutations in 16 patients with mucopolysaccharidosis type II (Hunter syndrome). Human Molecular Genetics type in (Hunter

10 Cleary MA, Wraith JE. Management of mucopolysaccharidosis type III. Arch Dis Child 1993; 69: 403-6.

11 McKusick VA. The mucopolysaccharidoses. Heritable disorders of connective tissue. 4th Ed. St Louis: Mosby, 1972: 598

12 Fong LV, Menahem S, Wraith JE, Chow CW. Endocardial fibroelastosis in mucopolysaccharidosis type VI. Clin Cardiol 1987; 10: 362-4.

13 Hayflick S, Rowe S, Kavanaugh-McHugh A, Olson JL Valle D. Acute infantile cardiomyopathy as a presentin feature of mucopolysaccharidosis VI. $\Im$ Pediatr 1992; 120 269-72.

14 Banna $M$, Hollenberg $R$. Compressive meningeal hypertrophy in mucopolysaccharidoses. AYNR 1987; 8: 385-6. 